\title{
Two in one: Unexpected acute pulmonary embolism detected by cardiovascular magnetic resonance in a patient with acute myocarditis
}

\author{
Magdalena Pływaczewska ${ }^{1}$, Dorota Piotrowska-Kownacka ${ }^{2}$, Olga Zdończyk ${ }^{1}$, \\ Marek Roik ${ }^{1}$, Krzysztof Jankowski ${ }^{1}$, Magdalena Koczaj-Bremer ${ }^{1}$, \\ Marek Gołębiowski ${ }^{2}$, Piotr Pruszczyk ${ }^{1}$
}

${ }^{1}$ Department of Internal Medicine and Cardiology, Medical University of Warsaw, Poland

${ }^{2} 1^{\text {st }}$ Department of Radiology, Medical University of Warsaw, Poland

Forty-year-old man with no record of cardiovascular diseases was admitted with suspected ST-segment elevation myocardial infarction (angina, elevated plasma troponin I level [36.13 ng/mL], ST-segment elevation in electrocardiogram leads I and $\mathrm{aVL}$ ), 3 days after a flu-like infection.

Patient underwent urgent coronary angiography which revealed normal arteries. Transtho- racic echocardiography showed left ventricular (LV) hipokinesis of lateral and apical segments with ejection fraction of $50 \%$. Due to suspected myocarditis cardiac magnetic resonance (CMR) was performed, which revealed myocardial edema (Fig. 1A, B) with corresponding late gadolinium enhancement typical for acute myocarditis involving up to $75 \%$ of the LV wall thickness (Fig. 1C, D).

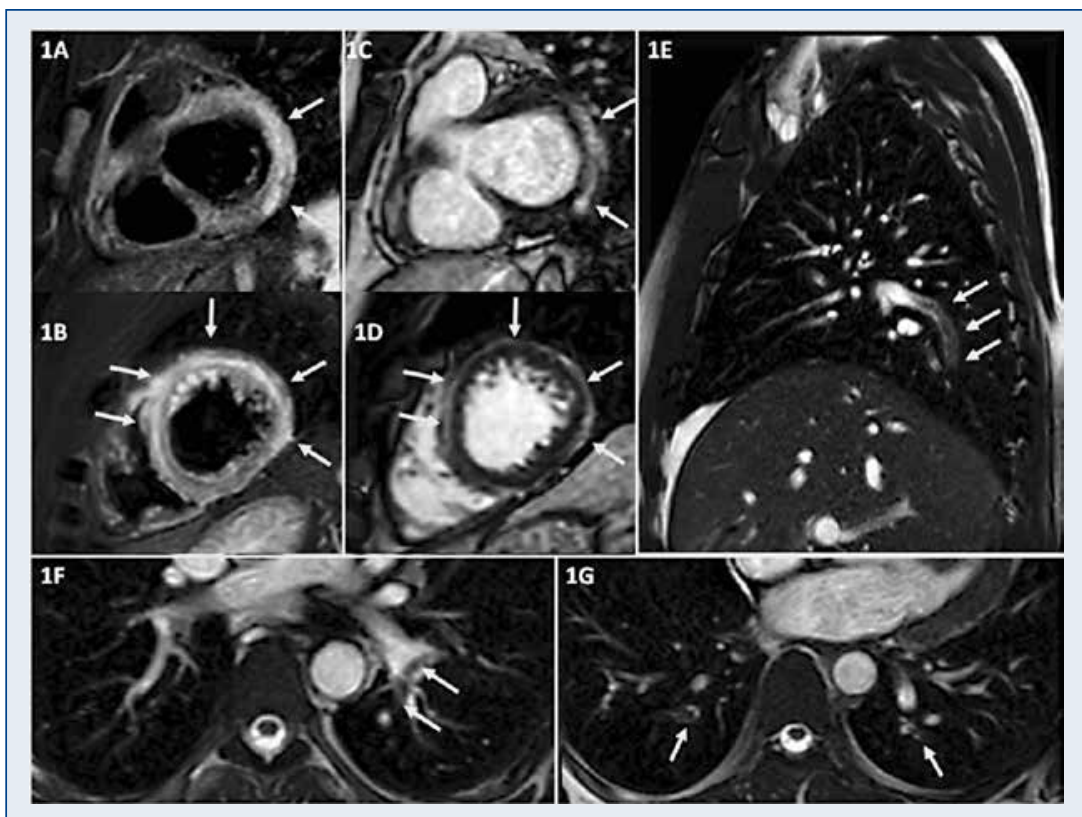

Figure 1. A, B. Myocardial edema (T2-weighted STIR black blood, short axis images); C, D. Myocardial late gadolinium enhancement of left ventricular walls; E. Thrombus partially occluding right lung lobe artery; F. Saddle thromboembolus at the bifurcation left lower lobe artery; G. Thrombus in segmental branches of left and right lower lobe arteries.

Address for correspondence: Magdalena Pływaczewska, MD, Department of Internal Medicine and Cardiology, Medical University of Warsaw, ul. Lindleya 4, 02-005 Warszawa, Poland, tel: +48 22 021144, fax: +48 225022142 , e-mail:ma.plywaczewska@gmail.com

Received: 25.09.2016 Accepted: 31.10.2016 
Additionally CMR demonstrated bilateral thromboemboli in lobar and segmental branches of pulmonary arteries (Fig. 1E, G). Anticoagulation with rivaroxaban was started. As antidsDNA, anticardiolipin and anti-beta2 glycoprotein antibodies were absent, antiphospholipid syndrome was excluded as a possible etiology of coexisting myocarditis and pulmonary embolism. Due to mildly reduced LV systolic function treatment with ramipril and carvedilol was introduced.

During months of follow up the patient was symptom free and in good general condition. Transthoracic echocardiography examination and the electrocardiography Holter monitoring were normal.
This reported case confirms high potential of CMR in the management of acute cardiovascular diseases. Currently, CMR has an established role in the diagnosis of myocardial infarction, myocarditis and aortic dissection.

Moreover, there is a growing evidence on CMR pulmonary angiography in the diagnosis of suspected pulmonary embolism.

However, still, CMR cannot be used as a first choice examination to verify acute pulmonary embolism suspicion due to lower sensitivity in detection of segmental and subsegmental thrombus compared to conventional computed tomographypulmonary angiography.

Conflict of interest: None declared 